\title{
Produção de Painéis Compensados Fenólicos com Lâminas de Madeira de Sequoia sempervirens
}

\author{
Setsuo Iwakiri ${ }^{1}$, Alexsandro Bayestorff da Cunha ${ }^{2}$, Rosilani Trianoski ${ }^{1}$, \\ Rafael Leite Braz ${ }^{3}$, Vinicius Gomes de Castro ${ }^{3}$, Sandra Kazmierczak ${ }^{3}$, \\ Edson Pinheiro ${ }^{3}$, Heloisa Rancatti ${ }^{3}$, Felipe Luis Sanches ${ }^{3}$
}

${ }^{1}$ Departamento de Engenharia e Tecnologia Florestal, Universidade Federal do Paraná - UFPR, Curitiba/PR, Brasil
${ }^{2}$ Departamento de Engenharia Florestal, Universidade Estadual de Santa Catarina - UDESC, Florianópolis/SC, Brasil
${ }^{3}$ Programa de Pós-graduação em Engenharia Florestal, Universidade Federal do Paraná - UFPR, Curitiba/PR, Brasil

\section{RESUMO}

Esta pesquisa teve como objetivo avaliar a qualidade dos painéis compensados multilaminados produzidos com lâminas de madeira de Sequoia sempervirens. Foram produzidos em laboratório compensados com cinco lâminas de 2,0 mm de espessura, coladas com resina fenol-formaldeído, com duas diferentes formulações: $(\mathrm{A}) \mathrm{FF}=100 ; \mathrm{E}=5 ; \mathrm{CC}=5 ; \mathrm{A}=5$, e (B) $\mathrm{FF}=100 ; \mathrm{E}=15$; $\mathrm{CC}=15 ; \mathrm{A}=15$, e duas gramaturas: $280 \mathrm{~g} / \mathrm{m}^{2}$ e $320 \mathrm{~g} / \mathrm{m}^{2}$. Os painéis foram prensados com pressão específica de $10 \mathrm{kgf} / \mathrm{cm}^{2}$, temperatura de $130{ }^{\circ} \mathrm{C}$ e tempo de prensagem de dez minutos. Foram avaliadas as propriedades de resistência da linha de cola aos esforços de cisalhamento e de flexão estática (módulo de elasticidade e módulo de ruptura, paralelo e perpendicular às fibras). De uma forma geral, as diferentes formulações de cola e gramaturas não afetaram significativamente as propriedades dos painéis, o que representa um aspecto importante sob o ponto de vista econômico. A resistência ao cisalhamento e a percentagem de falhas na madeira dos painéis produzidos com a formulação (B) atenderam aos requisitos mínimos da norma EN 314-2 (1993) para painéis de uso externo. Os resultados indicam que as lâminas de Sequoia sempervirens podem ser utilizadas para compor o miolo de painéis compensados para uso externo.

Palavras-chave: resina fenol-formaldeído, resistência da linha de cola, formulação de cola.

\section{Production of Phenolic Plywood Using Veneers of Sequoia sempervirens}

\begin{abstract}
The aim of this research was to evaluate the quality of plywood made from wood veneers of Sequoia sempervirens. Plywood with five $2.0 \mathrm{~mm}$ thick veneers were produced in laboratory and bonded with phenol-formaldehyde resinusing two different formulations: (A) resin $=100$, extender $=5$, filler $=5$, and water $=5$; and $(\mathrm{B})$ resin $=100$, extender $=15$, filler $=15$, and water $=15$, with two different amounts of glue spread: $280 \mathrm{~g} / \mathrm{m}^{2}$ and $320 \mathrm{~g} / \mathrm{m}^{2}$. The panels were pressed with specific pressure of $10 \mathrm{kgf} / \mathrm{cm}^{2}$, temperature of $130{ }^{\circ} \mathrm{C}$, and pressing time of 10 minutes. The properties of the glue line shear strengthand static bending (modulus of elasticity and modulus of rupture - parallel and perpendicular to the grain) were evaluated. In general, the different glue formulations and amounts of glue spread did not significantly affect the properties of the panels, which represent an important aspect from the economic point of view. The shear strengthand percentage of wood failures of the plywood produced withformulation (B) met the minimum requirements of the EN 314-2 (1993) standard to panels for external use. Results indicate that Sequoia sempervirens veneers can be used to compose the core of plywood for external use.
\end{abstract}

Keywords: phenol-formaldehyde resin, glue line strength, glue composition. 


\section{INTRODUÇÃO}

Estudos sobre espécies de rápido crescimento para produção de lâminas e compensados têm sido amplamente realizados no Brasil desde a década de 1990, com o objetivo de aumentar a quantidade de espécies alternativas ao pinus, que é a base da matériaprima para indústrias de painéis compensados.

Além das espécies do gênero Eucalyptus, vários pesquisadores têm estudado o comportamento de algumas espécies para produção de lâminas e compensados. Pinto \& Iwakiri (2013) avaliou o rendimento em laminação e qualidade dos painéis compensados de Criptomeria japônica; Bortoletto Junior \& Belini (2002) estudaram o comportamento da madeira de Schizolobium parayba para produção de compensados e Iwakiri et al. (2011) avaliaram a qualidade de painéis compensados produzidos com lâminas de Schizolobium amazonicum. Apesar da baixa densidade da madeira destas espécies, os pesquisadores encontraram resultados que indicam a sua potencialidade para produção de lâminas e compensados.

A Sequoia sempervirens (sequoia) é uma espécie nativa dos Estados Unidos da América e sua madeira apresenta cerne marrom avermelhado e alburno com tonalidade branca. A sua madeira, apesar da densidade baixa $\left(0,31 \mathrm{~g} / \mathrm{cm}^{3}\right)$, possui boa estabilidade dimensional e resistência à deterioração, além de outras características favoráveis para fabricação de painéis e produção de celulose (Diel \& Frizzo, 2002; Sloan \& Boe, 1974). Spichinger (2004) relata que a madeira de sequoia apresenta como características: boa usinabilidade e boa aderência para tintas e vernizes, além de não apresentar resinas, típicas de madeira de coníferas. Estas qualidades tornam a madeira de sequoia atrativa para a produção de painéis e o uso na indústria moveleira.

As limitações relacionadas à baixa densidade da madeira de espécies de florestas plantadas de rápido crescimento e aos pequenos diâmetros das árvores podem ser minimizadas com uso de tecnologias de colagem para fabricação de produtos de madeira reconstituída, como painéis laminados de madeira. Bendsten (1978) afirma que, para se alcançar êxito no uso dos recursos florestais, provenientes de florestas plantadas, os conceitos tradicionais devem ser modificados e adaptados às características da matéria-prima disponível.

$\mathrm{Na}$ produção de compensados, os fatores relacionados à tecnologia devem ser avaliados com muita atenção, principalmente quanto ao processo físico-químico envolvido na colagem de lâminas de madeira (Marra, 1992). O tipo de resina empregado na colagem deve ser adequado ao ambiente de utilização do painel, podendo ser ureia-formaldeído para ambiente interno e fenol-formaldeído para ambiente externo. A formulação da batida de cola e a gramatura são parâmetros que irão influenciar diretamente na qualidade da colagem e, ao mesmo tempo, no custo de produção do compensado (Baldwin, 1995; Sellers, 1993). Os autores afirmam que a densidade da madeira é um fator importante na definição destes parâmetros, tendo em vista as interações que ocorrem entre a porosidade da madeira e a absorção do adesivo na formação da ligação adesiva entre as lâminas. De acordo com Marra (1992), madeiras de baixa densidade absorvem maior quantidade de adesivo em função de sua maior porosidade; portanto, a viscosidade do adesivo deve ser aumentada para evitar a formação da linha de cola "faminta". Outros fatores, como pH e extrativos presentes na madeira, são também importantes, podendo interferir na cura do adesivo durante o processo de prensagem do painel na prensa quente. Segundo Marra (1992), o pH muito baixo de algumas espécies pode retardar a polimerização da resina fenol-formaldeído que cura no meio alcalino, reduzindo a resistência da ligação adesiva. Baldwin (1995) afirma que alguns extrativos presentes nas lâminas podem dificultar, durante a prensagem a quente, o processo de movimentação de água na forma de vapor de uma linha de cola para outra, destas para as bordas do painel, e posterior liberação para o ambiente externo. Este processo, sendo muito lento, terá como consequência o aumento da pressão interna de vapor, resultando no "estouro" no momento da abertura da prensa e da delaminação do painel.

Este trabalho teve como objetivo avaliar o potencial de uso da madeira de Sequoia sempervirens para produção de lâminas e painéis compensados para uso externo, com diferentes formulações e gramaturas do adesivo fenol-formaldeído. 


\section{MATERIAL E MÉTODOS}

Foi utilizada madeira de Sequoia sempervirens, com 18 anos de idade, em plantio experimental com espaçamento de $2,0 \times 2,0 \mathrm{~m}$, localizado na Estação Experimental da EPAGRI de São Joaquim, Estado de Santa Catarina. Para a laminação, foram utilizadas seis toras com comprimento de $0,60 \mathrm{~m}$, obtidas a partir de três árvores coletadas aleatoriamente no plantio. As toras foram descascadas e submetidas ao processo de aquecimento à temperatura em torno de $60{ }^{\circ} \mathrm{C}$, por período de 12 horas. As lâminas foram obtidas a partir de um torno piloto com espessura de $2,5 \mathrm{~mm}$ e seccionadas em uma guilhotina pneumática com dimensões de $600 \mathrm{~mm} \times 600 \mathrm{~mm}$. Os rendimentos de laminação foram determinados pela relação entre o volume da tora sem casca e após arredondamento e o volume das lâminas classificadas. A massa específica aparente e a massa específica básica da madeira foram determinadas em amostras retiradas de lâminas com dimensões de $20 \mathrm{~mm} \times 300 \mathrm{~mm}$.

Para a produção de painéis compensados, as lâminas foram secas em estufa com o teor de umidade médio de $6 \%$ e esquadrejadas em dimensões finais de $500 \mathrm{~mm} \times 500 \mathrm{~mm}$. Os painéis foram produzidos com cinco lâminas, utilizando-se a resina fenolformaldeído, com formulações de batida de cola com teores de sólidos de $42,60 \%$ e $37,72 \%$, e gramaturas de $280 \mathrm{~g} / \mathrm{m}^{2}$ e $320 \mathrm{~g} / \mathrm{m}^{2}$, conforme apresentado na Tabela 1. Os painéis foram prensados à temperatura de $130{ }^{\circ} \mathrm{C}$, pressão específica de $10 \mathrm{kgf} / \mathrm{cm}^{2}$ e tempo de permanência na prensa de dez minutos. Foram produzidos dois painéis por tratamento.

Após a prensagem, os painéis foram esquadrejados e acondicionados em câmara climática à temperatura de $20+3{ }^{\circ} \mathrm{C}$ e umidade relativa de $65+5 \%$, até sua estabilização. Para cada tratamento, foram retirados cinco corpos de prova para ensaios de flexão estática com a direção das fibras das lâminas da capa paralela ao comprimento do corpo de prova, e cinco corpos de prova na direção perpendicular. Para os ensaios de resistência da linha de cola aos esforços de cisalhamento, foram retirados dez corpos de prova para teste seco e dez para teste de fervura. Os ensaios foram conduzidos de acordo com as Normas Europeias EN 320 e EN 314, respectivamente. Na Figura 1, encontra-se
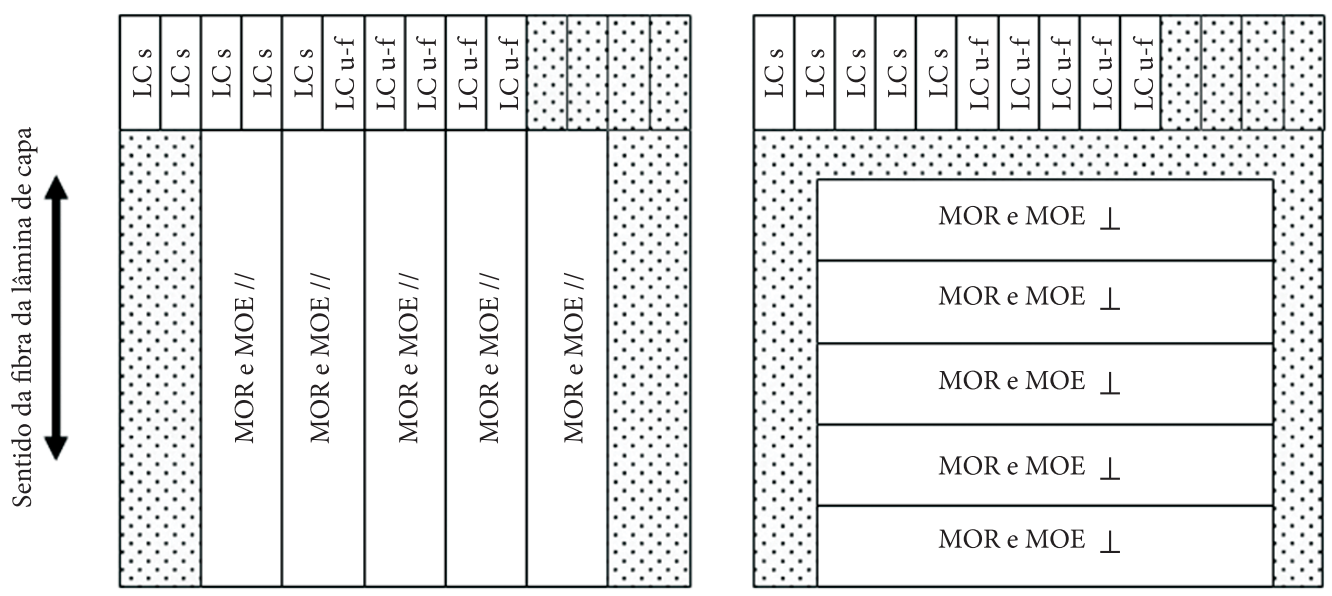

Figura 1. Representação do gabarito utilizado para retirada dos corpos de prova.

Figure 1. Representation of the template used for samples cutting.

Tabela 1. Delineamento experimental.

Table 1. Experimental design.

\section{Tratamento}

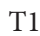

$\mathrm{T} 2$

T3

$\mathrm{T} 4$
Formulação

$A: F F=100 ; E=5 ; C C=5 ; A=5$

$A: F F=100 ; E=5 ; C C=5 ; A=5$

$\mathrm{B}: \mathrm{FF}=100 ; \mathrm{E}=15 ; \mathrm{CC}=15 ; \mathrm{A}=15$

$\mathrm{B}: \mathrm{FF}=100 ; \mathrm{E}=15 ; \mathrm{CC}=15 ; \mathrm{A}=15$
Gramatura $\left(\mathrm{g} / \mathrm{m}^{2}\right)$

280

320

280

320

FF: resina fenol-formaldeído; E: extensor - farinha de trigo; CC: casca de coco; A: água 
ilustrado o esquema de retirada de corpos de prova nos dois painéis de cada tratamento.

O delineamento estatístico utilizado foi o inteiramente casualizado e os resultados dos ensaios foram analisados por meio da Análise de Variância e do Teste de Tukey, no nível de probabilidade de 95\%.

\section{RESULTADOS E DICUSSÕES}

\subsection{Rendimento e qualidade das lâminas de madeira}

Os valores médios de massa específica aparente e massa específica básica da madeira de sequoia determinados a partir de lâminas foram, respectivamente, de $0,340 \mathrm{~g} / \mathrm{cm}^{3}$ e $0,290 \mathrm{~g} / \mathrm{cm}^{3}$. Os valores obtidos nesta pesquisa estão próximos do valor de massa específica básica citada por Marchiori (1996) de $0,310 \mathrm{~g} / \mathrm{cm}^{3}$ e de $0,348 \mathrm{~g} / \mathrm{cm}^{3}$ mencionado por Cown \& Mickney (2009), para madeira de sequoia com 38 anos de idade.

$\mathrm{O}$ rendimento total médio obtido para as seis toras laminadas foi de $41,21 \%$, com base no volume das toras sem casca, e o rendimento efetivo médio foi de $55,67 \%$, com base no volume das toras após o arredondamento. Esta diferença entre os rendimentos pode ser atribuída ao alto grau de conicidade das toras. As avaliações da qualidade das lâminas demonstraram a presença de nós em $100 \%$ das lâminas, sendo um indicativo de que as árvores destes plantios experimentais não passaram por procedimentos de podas. Foram observadas também presença de lâminas com rugosidades e fendas superficiais.
Os resultados de rendimento obtidos nesta pesquisa foram próximos daqueles encontrados por outros autores, para espécies de pinus e eucaliptos provenientes de plantios florestais. Almeida et al. (2004) obtiveram, para clones I e II de Eucalyptus grandis $\times$ Eucalyptus urophylla, rendimento médio de $51,74 \%$ e $56,81 \%$, respectivamente. Pio (1996) encontrou, para espécies de Eucalyptus scabra e Eucalyptus robusta, rendimento médio de $36,47 \%$ e $44,00 \%$, respectivamente. O autor cita ainda, como fontes de referências, os rendimentos médios para as seguintes espécies de pinus: Pinus elliottii - 42,40\%, Pinus taeda - 55,50\% e Pinus strobus - 54,40\%. Interanmense (1998) obteve para espécies de E. viminalis, E. cloeziana e E. maculatavalores valores médios de rendimento de $50,00 \%, 50,43 \%$ e $44,86 \%$, respectivamente.

\subsection{Resistência da linha de cola às tensões de cisalhamento}

Os valores médios de resistência da linha de cola e percentagem de falhas na madeira dos painéis estão apresentados na Tabela 2.

Os resultados dos ensaios de cisalhamento da linha de cola no teste seco indicam que houve influência significativa da formulação da batida de cola apenas nos painéis produzidos com gramatura de $280 \mathrm{~g} / \mathrm{m}^{2}$. Os painéis produzidos com menor teor de sólidos (formulação B - T3) apresentaram média estatisticamente superior em relação aos painéis produzidos com a formulação B (T1), com maior teor de sólidos. Este resultado contraria a teoria qualitativa sobre a colagem, tendo em vista que a formulação (A) possui maior percentagem de resina fenólica na sua composição. $\mathrm{O}$ aumento na gramatura

Tabela 2. Resultados dos ensaios de cisalhamento da linha de cola dos painéis compensados de Sequoia sempervirens. Table 2. Results of the glue line shear tests of the Sequoia simpervirens playwood.

\begin{tabular}{|c|c|c|c|c|c|c|}
\hline \multirow[b]{2}{*}{ Tratamento } & \multicolumn{3}{|c|}{ Teste seco } & \multicolumn{3}{|c|}{ Teste fervura } \\
\hline & $\begin{array}{l}\text { Média } \\
(\mathrm{MPa})\end{array}$ & $\begin{array}{l}\text { CV } \\
(\%)\end{array}$ & $\begin{array}{l}\text { FM } \\
(\%)\end{array}$ & $\begin{array}{l}\text { Média } \\
\text { (MPa) }\end{array}$ & $\begin{array}{l}\text { CV } \\
(\%)\end{array}$ & $\begin{array}{l}\text { FM } \\
(\%)\end{array}$ \\
\hline $\mathrm{T} 1$ & $1,13^{\mathrm{b}}$ & 20,60 & 43 & $0,66^{b}$ & 27,18 & 38 \\
\hline $\mathrm{T} 2$ & $1,30^{\mathrm{ab}}$ & 8,70 & 60,9 & $0,94^{\mathrm{a}}$ & 23,14 & 31,1 \\
\hline T3 & $1,37^{\mathrm{a}}$ & 10,58 & 52 & $0,81^{\mathrm{ab}}$ & 38,19 & 71,3 \\
\hline $\mathrm{T} 4$ & $1,24^{\mathrm{ab}}$ & 18,82 & 59,7 & $0,92^{\mathrm{ab}}$ & 12,56 & 73 \\
\hline
\end{tabular}

Médias seguidas pela mesma letra não diferem estatisticamente entre si, a 95\% de probabilidade. CV: coeficiente de variação; FM: falha na madeira. 
de $280 \mathrm{~g} / \mathrm{m}^{2}$ para $320 \mathrm{~g} / \mathrm{m}^{2}$ não influenciou de forma positiva a resistência da linha de cola dos painéis no teste seco.

Para o teste de fervura, não foram constatadas diferenças estatisticamente significativas entre os painéis produzidos com diferentes formulações da batida de cola $(\mathrm{T} 1 \times \mathrm{T} 3$ e $\mathrm{T} 2 \times \mathrm{T} 4)$. A igualdade estatística entre os resultados obtidos indica a possibilidade de colagem de painéis com menor teor de sólidos da batida de cola (formulação B), o que representa redução nos custos de produção. Em relação à gramatura, constatou-se diferença significativa apenas para os painéis produzidos com $280 \mathrm{~g} / \mathrm{m}^{2}$, sendo que o aumento na gramatura para $320 \mathrm{~g} / \mathrm{m}^{2}$ resultou em maior valor médio de resistência ao cisalhamento.

Em relação aos resultados apresentados na literatura, os valores médios de resistência ao cisalhamento no teste de fervura obtidos nesta pesquisa, entre 0,66 e 0,94 MPa, estão abaixo dos valores médios encontrados por Iwakiri et al. (2002) para painéis compensados de Pinus taeda produzidos com resina fenol-formaldeído, situados entre 0,94 e 1,26 MPa. Por outro lado, Bortoletto Junior (2003) obteve, para painéis compensados fenólicos produzidos com 11 espécies de eucalipto, resistência da linha de cola no teste de fervura na faixa de 1,91 a 2,12 $\mathrm{MPa}$, porém com percentagem de falhas na madeira abaixo de $20 \%$. Os menores valores de resistência ao cisalhamento obtidos nesta pesquisa podem ser atribuídos à baixa densidade da madeira de sequoia $\left(0,340 \mathrm{~g} / \mathrm{cm}^{3}\right)$, que contribuiu para maior absorção do adesivo, resultando na redução da espessura da linha de cola e, consequentemente, da resistência da ligação adesiva entre as lâminas.
Na avaliação comparativa com a Norma Europeia EN 314-2 (1993), todos os tratamentos apresentaram, para o teste seco, valores médios de resistência ao cisalhamento superiores ao valor mínimo de 1,0 $\mathrm{MPa}$, independentemente de percentagem de falhas na madeira. Diversamente, para o teste de fervura, apenas os painéis produzidos com a formulação $B$ (T3 e T4) atingiram o requisito mínimo da norma na referência que estabelece valores de resistência ao cisalhamento entre 0,6 e 1,0 $\mathrm{MPa}$, associados à percentagem de falhas na madeira superior a $40 \%$.

\subsection{Flexão estática paralela}

$\mathrm{Na}$ Tabela 3, estão apresentados os valores médios de massa específica, módulo de elasticidade (MOE//) e módulo de ruptura (MOR//) paralelo dos painéis compensados de madeira de sequoia.

Para estas amostras de painéis, os valores médios de massa específica variaram de 0,394 a $0,419 \mathrm{~g} / \mathrm{cm}^{3}$.

Tanto para o MOE// quanto para o MOR//, não foram constatadas diferenças estatisticamente significativas entre os painéis produzidos com diferentes formulações de batida de cola e gramaturas. Também em termos de médias absolutas, não se podem observar correlações diretas sobre os efeitos destes dois parâmetros do processo produtivo nas propriedades dos painéis avaliados. Estes resultados indicam vantagens em termos econômicos, tendo em vista a possibilidade de redução no consumo de cola.

Os resultados de MOE// e MOR// obtidos para os painéis compensados de sequoia foram inferiores quando comparados com os valores obtidos por Iwakiri et al. (2002) para painéis de Pinus taeda, cujos valores médios foram, respectivamente, de 5.800 MPa e 58,4 MPa. As diferenças observadas

Tabela 3. Resultados de massa específica e flexão estática paralela dos painéis compensados de Sequoia sempervirens. Table 3. Results of the density and parallel static bending of the Sequoia semperviren splywood.

\begin{tabular}{|c|c|c|c|c|c|c|}
\hline \multirow{2}{*}{ Tratamento } & \multicolumn{2}{|c|}{$\operatorname{ME}\left(\mathrm{g} / \mathrm{cm}^{3}\right)$} & \multicolumn{2}{|c|}{ MOE// (MPa) } & \multicolumn{2}{|c|}{ MOR// (MPa) } \\
\hline & Média & CV (\%) & Média & CV (\%) & Média & CV (\%) \\
\hline $\mathrm{T} 1$ & 0,415 & 2,76 & $3.289^{\mathrm{a}}$ & 8,28 & $29^{\mathrm{a}}$ & 17,52 \\
\hline $\mathrm{T} 2$ & 0,419 & 1,93 & $3.651^{\mathrm{a}}$ & 6,78 & $24^{\mathrm{a}}$ & 20,48 \\
\hline T3 & 0,394 & 2,88 & $3.188^{\mathrm{a}}$ & 9,35 & $26^{\mathrm{a}}$ & 19,25 \\
\hline $\mathrm{T} 4$ & 0,414 & 3,59 & $3.624^{\mathrm{a}}$ & 18,81 & $28^{\mathrm{a}}$ & 9,96 \\
\hline
\end{tabular}

Médias seguidas pela mesma letra não diferem estatisticamente entre si, a 95\% de probabilidade. ME: massa específica; MOE//: módulo de elasticidade; MOR//: módulo de ruptura; CV: coeficiente de variação. 
Tabela 4. Resultados de massa específica e flexão estática perpendicular dos painéis compensados de Sequoia sempervirens.

Table 4. Results of density and perpendicular static bending of the Sequoia semperviren splywood.

\begin{tabular}{|c|c|c|c|c|c|c|}
\hline \multirow{2}{*}{ Tratamento } & \multicolumn{2}{|c|}{$\operatorname{ME}\left(\mathrm{g} / \mathrm{cm}^{3}\right)$} & \multicolumn{2}{|c|}{$\mathrm{MOE}^{\perp}(\mathrm{MPa})$} & \multicolumn{2}{|c|}{$M O R \perp(M P a)$} \\
\hline & Média & CV (\%) & Média & CV (\%) & Média & CV (\%) \\
\hline $\mathrm{T} 1$ & 0,415 & 3,26 & $1.317^{\mathrm{a}}$ & 11,17 & $16^{\mathrm{a}}$ & 23,16 \\
\hline $\mathrm{T} 2$ & 0,426 & 3,36 & $1.094^{\mathrm{a}}$ & 8,98 & $12^{\mathrm{a}}$ & 21,57 \\
\hline T3 & 0,423 & 4,82 & $1.014^{\mathrm{a}}$ & 9,73 & $13^{\mathrm{a}}$ & 46,82 \\
\hline $\mathrm{T} 4$ & 0,408 & 6,98 & $1.151^{\mathrm{a}}$ & 4,11 & $15^{\mathrm{a}}$ & 10,87 \\
\hline
\end{tabular}

Médias seguidas pela mesma letra não diferem estatisticamente entre si, a 95\% de probabilidade. ME: massa específica; MOE ${ }^{\perp}$ : módulo de elasticidade; $M \mathrm{R}^{\perp}$ : módulo de ruptura; $\mathrm{CV}$ : coeficiente de variação.

podem ser atribuídas à menor massa específica dos painéis de sequoia, entre 0,394 e $0,419 \mathrm{~g} / \mathrm{cm}^{3}$, em relação aos painéis de Pinus taeda com massa específica de $0,500 \mathrm{~g} / \mathrm{cm}^{3}$. Em relação aos painéis comerciais de Pinus taeda, os dados apresentados no catálogo técnico da ABIMCI (2002) são de $6.890 \mathrm{MPa}$ e $38,1 \mathrm{MPa}$, respectivamente, para o $\mathrm{MOE} / /$ e $\mathrm{MOR} / /$, para painéis de massa específica de $0,53 \mathrm{~g} / \mathrm{cm}^{3}$.

\subsection{Flexão estática perpendicular}

Na Tabela 4, estão apresentados os valores médios de massa específica, módulo de elasticidade $\left(\mathrm{MOE}^{\perp}\right)$ e módulo de ruptura $\left(\mathrm{MOR}^{\perp}\right)$ perpendicular dos painéis compensados de sequoia.

Para estas amostras de painéis, os valores médios de massa específica variaram na faixa de 0,408 a $0,426 \mathrm{~g} / \mathrm{cm}^{3}$.

Tanto para o MOE quanto para o MOR perpendicular, não foram constatadas diferenças estatisticamente significativas entre os painéis produzidos com diferentes formulações de batida de cola e gramaturas. Da mesma forma, como nos ensaios de flexão paralelos, em termos de médias absolutas, não se podem constatar correlações diretas sobre os efeitos destes dois parâmetros do processo produtivo nas propriedades dos painéis avaliados, o que indica vantagens econômicas resultantes da redução no consumo de cola.

Os resultados de $\mathrm{MOE}^{\perp}$ e $\mathrm{MOR}^{\perp}$ obtidos para os painéis compensados de sequoia foram inferiores em comparação aos painéis comerciais de Pinus taeda, cujos valores foram de $2.839 \mathrm{MPa}$ e 25,3 MPa, respectivamente. Essas diferenças podem ser atribuídas à menor massa específica dos painéis de sequoia em relação à massa específica dos painéis comerciais de Pinus taeda, de $0,530 \mathrm{~g} / \mathrm{cm}^{3}$.

\section{CONCLUSÕES}

Os resultados de rendimento em laminação obtidos para Sequoia sempervirens foram próximos daqueles encontrados pelos outros autores para espécies de pinus e eucaliptos provenientes de plantios florestais.

De uma forma geral, as diferentes formulações de cola e gramaturas não afetaram significativamente as propriedades dos painéis compensados, o que representa um aspecto importante sob o ponto de vista econômico.

A resistência ao cisalhamento e a percentagem de falhas na madeira dos painéis produzidos com a formulação B atenderam aos requisitos mínimos da norma EN 314-2 (1993) para painéis de uso externo.

Os resultados indicam que as lâminas de Sequoia sempervirens podem ser utilizadas para compor o miolo de painéis compensados para uso externo, com a possibilidade de colagem de forma associada com lâminas de espécies com maior densidade e resistência mecânica nas capas.

\section{AGRADECIMENTOS}

Os autores expressam seus agradecimentos à EPAGRI - Estação Experimental de São Joaquim - SC, pela doação da madeira de Sequoia sempervirens utilizada nesta pesquisa.

\section{STATUS DA SUBMISSÃO}

Recebido: 24/01/2012

Aceito: 24/01/2013

Publicado: 30/06/2013 


\section{AUTOR(ES) PARA CORRESPONDÊNCIA}

\section{Setsuo Iwakiri}

Departamento de Engenharia e

Tecnologia Florestal - DETF,

Universidade Federal do Paraná - UFPR,

Av. Lothário Meissner, 632, Jardim Botânico,

CEP 23890-000, Curitiba, PR, Brasil

e-mail: setsuo@ufpr.br

\section{Rosilani Trianoski}

Departamento de Engenharia e

Tecnologia Florestal - DETF,

Universidade Federal do Paraná - UFPR,

Av. Lothário Meissner, 632, Jardim Botânico,

CEP 23890-000, Curitiba, PR, Brasil

e-mail: rosillani@ufpr.br

\section{REFERENNCIAS}

Associação Brasileira da Indústria da Madeira Processada Mecanicamente - ABIMCI. Catálogo técnico de compensados de pinus. Curitiba; 2002. 20 p.

Almeida RR, Bortoletto Junior G, Jankoski IP. Produção de lâminas a partir da madeira de clones do híbrido Eucalyptus grandis $x$ Eucalyptus urophylla. ScientiaForestalis 2004; 65: 49-58.

Baldwin RF. Plywood and veneer-based products: manufacturing practices. San Francisco: Miller Freeman; 1995.

Bendsten BA. Properties of wood from improved and intensively managed tress. In: Proceedings of the economics and financial management and timber production technical comitees of the forest products research society; 1978; Atlanta. Atlanta; 1978.78 p.

Bortoletto Junior G. Produção de compensados com 11 espécies do gênero Eucalyptus, avaliação das suas propriedades físico-mecânicas e indicações para utilização. ScientiaForestalis 2003; 63: 65-78.

Bortoletto Junior G, Belini UL. Produção de lâminas e manufatura de compensados a partir da madeira de guapuruvu (Schizolobium parahyba Blake) proveniente de um plantio misto de espécies nativas. Cerne 2002; 8(2): 1-16.

Cown DJ, Mckiney RB. Wood properties of 38 year-old redwood from Mangatu Forest. New Zealand Journal of Forestry 2009; 54(2): 25-32.
European Committee for Standardization. EN 310: Plywood - Determination of modulus of elasticity and modulus of rupture in static bending. EN; 1993.

European Committee for Standardization. EN 314-2: Plywood - Determination of shear bonding strength. EN; 1993.

Inteteramnense MT. Utilização das madeiras de Eucalyptus cloeziana (F. Muell), Eucalyptus maculata (Hook) e Eucalyptus puctata DC var. punctata para produção de painéis compensados [dissertação]. Curitiba: Setor de Ciências Agrárias, Universidade Federal do Paraná; 1998.

Iwakiri S, Del Menezzi CHS, Laroca, C, Venson I, Matoski, S.S. Produção de compensados de Pinus taedae Pinus oocarpa com resina fenol-formaldeído. Cerne 2002; 11(2): 092-097.

Iwakiri S, Vargas CA, Parchen CFA, Weber, C, Batista CC, Garbe EA et al. Avaliaçao da qualidade de painéis compensados produzidos com lâminas de madeira de Schizolobium amazonicum. Floresta 2011; 41(3): 451-458.

Diel J, Fizzo S. Estudos de caracterização da Sequóia sempervirens para produção de celulose Kraft. In: Anais do $35^{\circ}$ Congresso e exposição anual de celulose e papel; 2002; São Paulo. São Paulo; 2002.

Marchiori JNC. Dendrologia das Gimnospermas. Santa Maria: Editora UFSM; 1996.

Marra AA. Technology of wood bonding: principles in practice. New York: Van Nostrand Reinhold; 1992.

Pinto JA, Iwakiri S. Estudo sobre a viabilidade do uso da madeira de "Cryptomeria japonica" para produção de painéis compensados. Scientia Forestalis 2013; 41(97): 29-37.

Pio NS. Avaliação da madeira de Eucalyptus scabra (Dum-Cours) e Eucalyptus robusta (Smith) na produção de painéis compensados [dissertação]. Curitiba:Setor de Ciências Agrárias, Universidade Federal do Paraná; 1996.

Sellers T. Plywood and adhesive technology. New York: Marcel Dekker; 1993.

Sloan J, Boe K. Sequoia sempervirens (Lamb. Ex D. Don) Endl. In: Seeds of Woody plants in the United States; 1974; Schopmeyer, USA. Schopmeyer; 1974.

Spichinger OA. Aprovechamiento em el aserrado de sequoia (Sequoia sempervirens (D. DON) Endl.) y classificación de la madeira obtenida [dissertação]. Santiago do Chile: Departamento de Ingeniería de La madeira, Universidad de Chile; 2004. 